\title{
Femur Bone Stress Analysis in CFD Modules with Parallel Processing
}

\author{
Houneida Sakly, Mourad Said, Moncef Tagina
}

\begin{abstract}
This research focuses on the aspect of femur bone modeling that will change structure in response to mechanical stresses that can be induced to bone formation. $3 D$ femur bone models are constructed by configuring material and geometric conditions and respecting patients' specific features, providing realistic and performant structural analysis. Using CFD concept, a three-dimensional model of the femur system was established, calculated the level of stress, the distribution of the femur and the magnitude of the transmitted force. This study describes a $3 D$ construction process as well as the generation of the mesh based on a parallel processing of eight processors. Our main contribution revolves around the use of CFD modules to simulate stress measurement in the bone and study their impact in the case of external force exerted with successive values $10 \mathrm{~N}, 50 \mathrm{~N}$ and $100 N$.
\end{abstract}

Keywords : 3D Femur bone, geometric conditions, CFD Module, Stress

\section{INTRODUCTION}

$\mathrm{B}_{\text {one diseases influence the quality of life of patients }}$ after the age of 50 . The process of bone remodeling induces a deterioration of bone quality, leading to an increased risk of exposure. The mortality rate of patients with osteoporotic fractures is 15 to $30 \%$ following medical antecedents such as infections, venous thrombosis and cardiovascular disease [1] [2] [3]. Bone is considered a material of functional caliber. It consists of the following components: hydroxyapatite, collagen, traces of proteoglycans, non-collagenous proteins and water[4]. According to most measurements, the femur bone (Fig.1) is estimated as the longest and strongest bone in the human body [5].

Revised Manuscript Received on December 30, 2019.

* Correspondence Author

Houneida Sakly*, COSMOS Laboratory -National Institute of Computer Sciences - Campus University of Mannouba -Tunisia. Email: houneida.sakly@esiee.fr

Mourad Said, Radiology and Medical Imaging Unit, International Center Carthage Medical, Tourist Area "JINEN EL OUEST"-5000 Monastir, Tunisia. Email: mouradsaid@yahoo.fr

Moncef Tagina, COSMOS Laboratory -National Institute of Computer Sciences - Campus University of Mannouba -Tunisia. Email: moncef.tagina@ensi-uma.tn

(C) The Authors. Published by Blue Eyes Intelligence Engineering and Sciences Publication (BEIESP). This is an open access article under the CC BY-NC-ND license (http://creativecommons.org/licenses/by-nc-nd/4.0/)

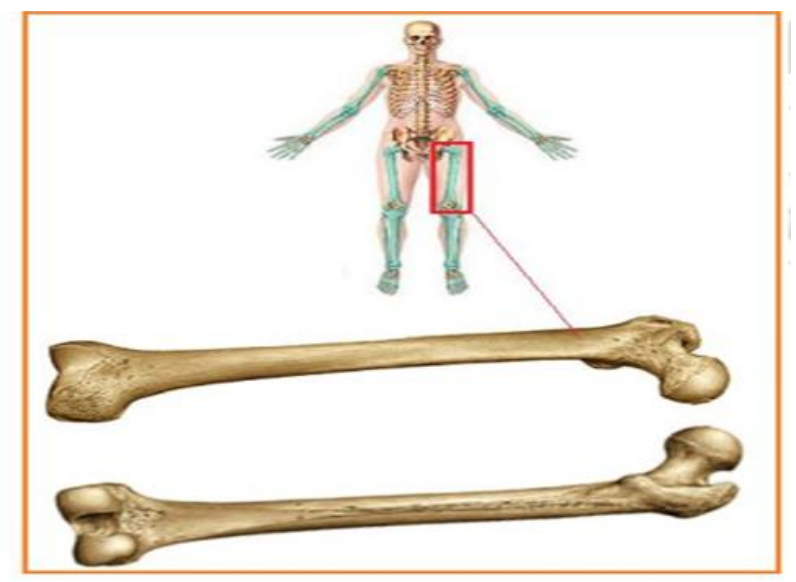

Fig.1.The femur Bone [5]

The average of its length is assessed at $26.74 \%$ compared to the size of a normal person [2]. The neck has a length of 40 to $50 \mathrm{~mm}$. Its diameter is considered the smallest front-back as well as it is compressed in its middle. The measurements of the angle is not stable: for children, it is about $150 \mathrm{o}$ and for old people at $120 \mathrm{o}$ on average. Given the strength of femur bone and for a fracture to occur, it must be applied a significant force. In particular, in patients with normal bone resistance, car accidents or falling from a height are considered among the crucial causes for having a fracture. In this paper, we will exploit the CFD modules to simulate the value of stress in case of the presence of external force exerted with successive value $10 \mathrm{~N}, 50 \mathrm{~N}, 100 \mathrm{~N}$.

\section{METHODS AND MATERIALS}

A 3D model for femur bone with $114.2 \mathrm{k}$ cells and $20.5 .3 \mathrm{k}$ nodes was performed. A Tet-Dominant Algorithm was executed for the quick generation and automated tetrahedral meshes. Manual meshing size was setting with minimum edge length $=0.001 \mathrm{~m}$ and maximum edge length $=0.003 \mathrm{~m}$, moderate quality and second order for the solver multifrontal as well as 8 processors for parallel processing and maximum runtime $=3600$ s.

\section{RESULTS AND DISCUSSION}

The femur bone was tested under three axes of translation in the nodal directions $\mathrm{x}, \mathrm{y}$ and $\mathrm{z}$ and rotations around the nodal directions $\mathrm{x}, \mathrm{y}$ and $\mathrm{z}$ (fig.2). A Tet-Dominant Algorithm was executed for the quick generation and automated tetrahedral meshes is detailed systematically as shown in Table.1: 
Table-I:Steps of the Tet-Dominant Algorithm

\section{Step1: Initialization}

BC $=[\text { None }]^{*}$; $/ /$ Initialize list for boundary conditions

Femur bone // Initialize list for materials

FC $=[\text { None }]^{*} 1 ; / /$ Initialize list for field calculations

\section{Step2: mesh generation \\ MESH=Read Mesh( \\ UNITE $=20$, \\ FORMAT='MED', \\ ); // Define mesh file}

\section{Step3: 3D mesh Model}

MODEL=AFFE_MODELE(
Model=MESH,
MODELISATION='3D',), // Model definition of phenomena and
element types

Step4: setting the force (boundary condition)

BC[2]=AFFE_CHAR_MECA(

MODELE=MODEL,

FORCE_FACE $=$ F (

$\mathbf{F X}=((0.0) /($ areaDict['faceGroupOnGeoFaces_8']+areaDict['faceGroup OnGeoFaces_10']+areaDict['faceGroupOnGeoFaces_9'])),

// Example of boundary condition: force (along the $\mathrm{X}$ axis)

Step5: linear solver and statistical analysis

SOLVER $=$ F $F($

STOP SINGULIER='True',

METHODE='MULT_FRONT',

RENUM='MDA',

NPREC $=8$, ) // Linear static analysis definition

SIM=CALC_CHAMP(

GROUP_MA=('volumeOnGeoVolumes_0' $)$,

reuse $=$ SIM,

DEFORMATION=('EPSI_NOEU'),

CRITERES=('SIEQ_NOEU'),

RESULTAT $=$ SIM,

) // Derived result calculation on nodes

\section{Step6: implementation of the solution}

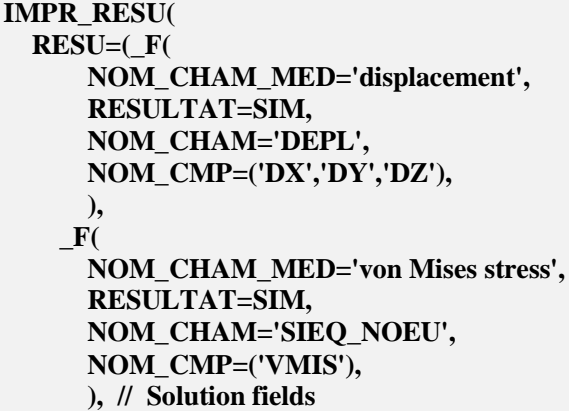

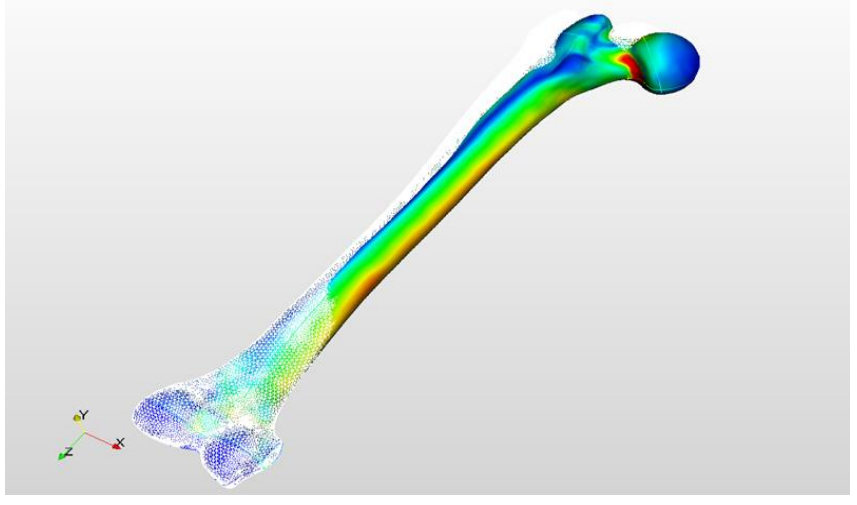

Fig.2.3D Femur bone

The generation of the mesh presented in Fig.3 ended with 35459 points, 140381 elements, badmax edge $=5.72985 \mathrm{e}+$ 12, 37 splits performed and total badness $=224290$.

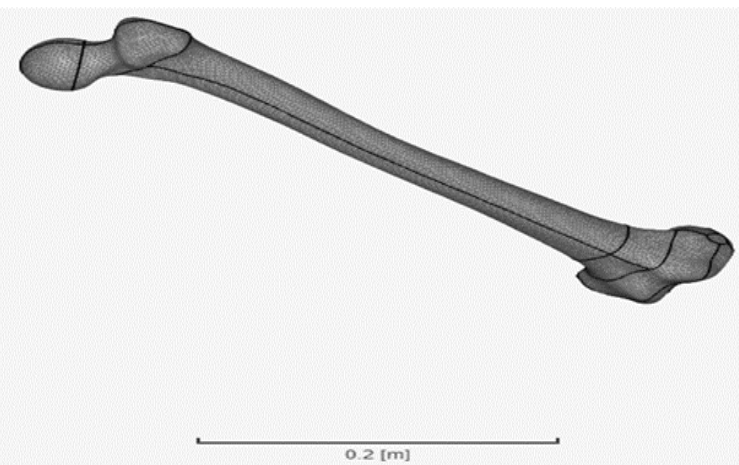

Fig.3.Mesh of the Femur bone

For a given load, the external surface is subjected to high stresses because the bending of the bones is more stressed for pure axial compression. As a result, recent research work shows that bending bones have lower mechanical strengths than bones subjected to pure axial compression [6] [7]. By analogy the two resulting forces, $R_{h}$ and $R_{t}$ are solicited by femur bone ,the resulting trochanteric force $R_{t}$ is the vector sum of the resultant muscle force $F_{m t}$ exerted by all the abdominal muscles and the force $F_{m k}$. In order to properly simulate the loading condition of the bone, knowledge of the biomechanics involved is required.

In our case, the following parameter for the stress analysis was chosen: linear elastic for the material behavior and the isotropic directional dependency model. The Young's modulus $=15000000000(\mathrm{~Pa})$ which characterizes the stiffness of the materiel ,the Poisson's ratio $=0.22$ which describes the compression of the materiel transverse to the axial stain and the density $=1500 \mathrm{~kg} / \mathrm{m}^{3}$ which defines the relationship between it masses and the space occupied by it [10] [8] [9] [11] [12]. The matrix is of size is composed of 616044 equations. It contains 22732539 non-zero terms if it is symmetric and 44849034 non-zero terms if it is not symmetric (the number of non-zero terms is likely to vary if one uses the contact in continuous formulation or method XFEM with contact). That is to conclude a rate of filling of $0.012 \%$.A summary of the results is mentioned in table. 2 :

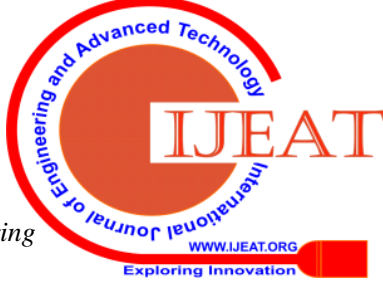


Table-II:summary table for the results

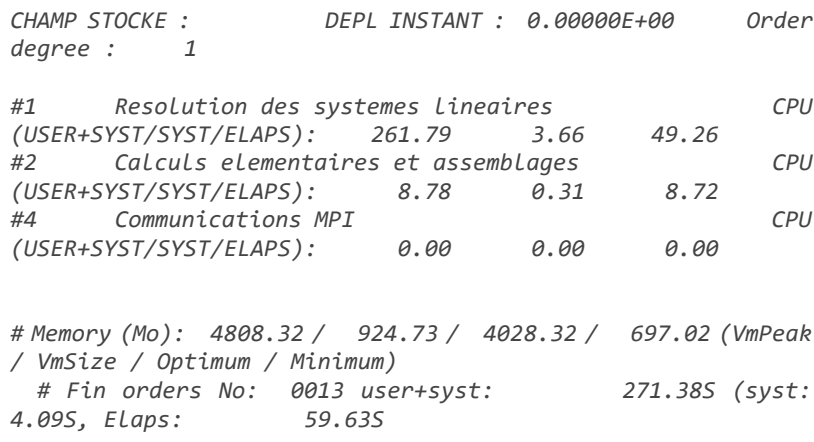

The distribution of stresses in the femur bone is shown in Figure.4. The allowable bending force was $10 \mathrm{~N}, 100 \mathrm{~N}$, and $500 \mathrm{~N}$ simultaneously in the 3 stress analysis attempts.

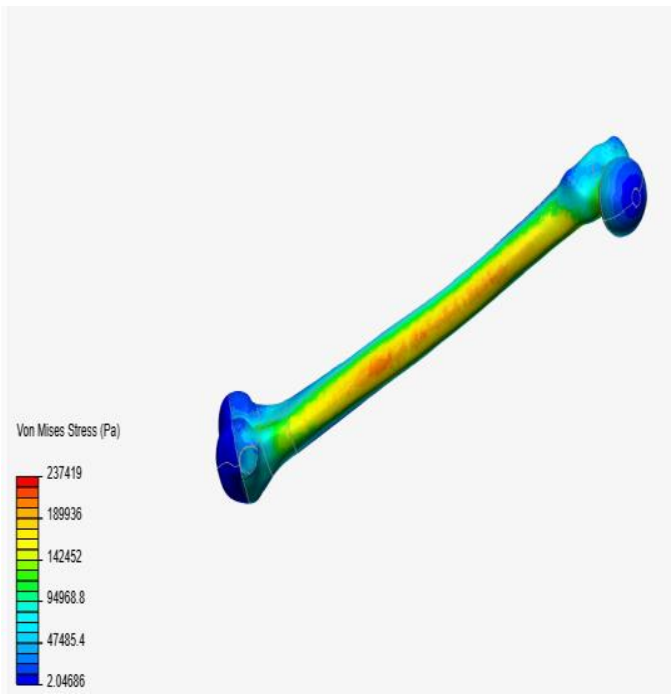

(A)Stress simulation with bending Force $=50 \mathrm{~N}$

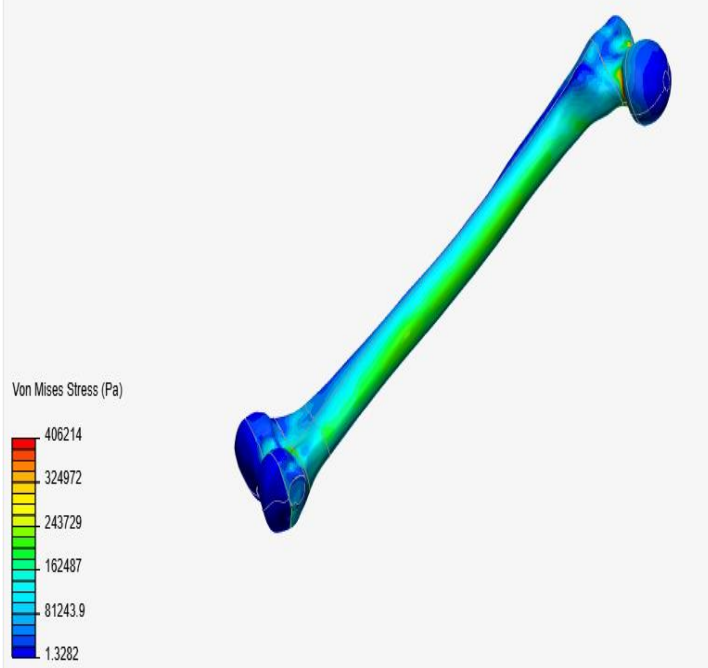

(B)Stress simulation with bending Force $=100 \mathrm{~N}$

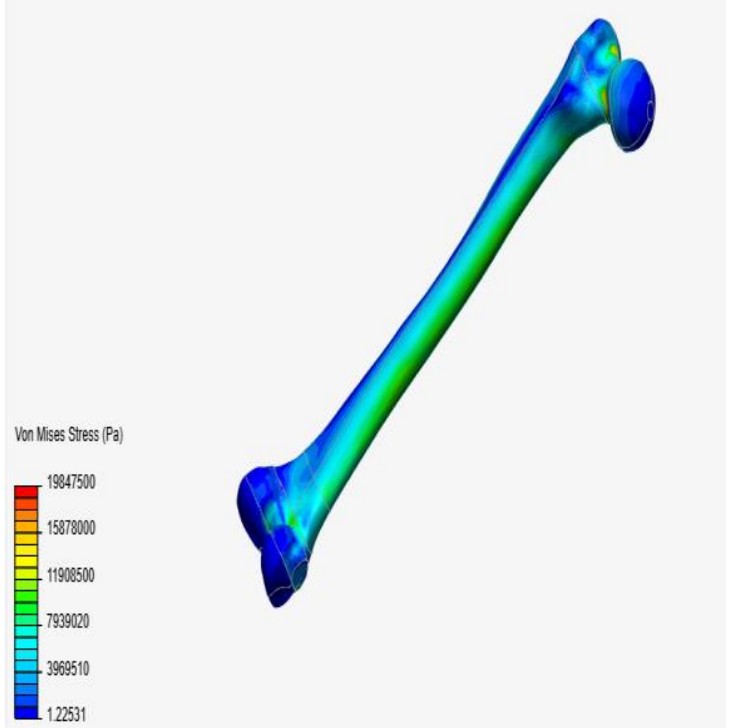

(c)Stress simulation with bending Force $=500 \mathrm{~N}$

Fig.4.Stress Simulation (A) Stress simulation with bending Force $=50 \mathrm{~N}$ (B) Stress simulation with bending Force $=100 \mathrm{~N}$ (c) Stress simulation with bending Force $=500 \mathrm{~N}$

The stress analysis for the three attempt occupies a mean total of CPU Time $=286.05 \mathrm{~s}$, a mean CPU time for a total use $=280.30 \mathrm{~S}$ and a an average CPU time for the remaining 35995213.95 s. The displacement of the velocity stress in the femur bone is estimated around $4 \mathrm{e}+6(\mathrm{~N} / \mathrm{m} 2)$.

Therefore, what is perceived as a result that the force exerted on the bone could give interesting indications on stress measurements in the femur bone as well as a velocity displacement vector that increases viewing the stress pressure

\section{CONCLUSION}

In conclusion, this study highlights the advantages of CFD models specific to the reconstruction of 3D femur models. The evaluation of fracture risk and the optimization of surgical intervention depends on the bone density, mechanical properties and geometry. As a result, there is no alternative to experimental mechanical testing to consider all of these parameters when determining bone failure loads. The modification of the values of the force exerted on the bone simulates the undesired lateral impacts, such as those occurring during unforeseen accidents. The modeling with CFD for the analysis of stress was interesting to study the impact of the external force compared to the resistance of bone. The simulation of the stress measurement in the bone and the analysis of their impact in the case of external force exerted with successive values $10 \mathrm{~N}, 50 \mathrm{~N}$ and $100 \mathrm{~N}$ can lead to a promising medical decision support.

\section{ACKNOWLEDGMENT}

Special recognition addressed to the Carthage International Medical Center that supported this work and to the medical staff for providing us with an access to the patients' archive and the administrative framework for the warm welcome in their team.

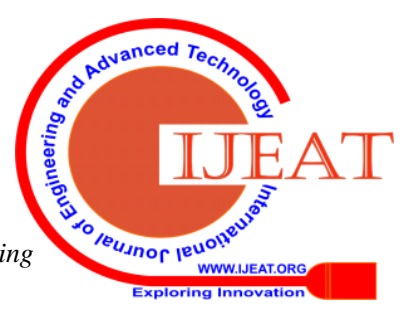




\section{REFERENCES}

1. M. T. Bahia, M. B. Hecke, and E. G. F. Mercuri, "Image-based anatomical reconstruction and pharmaco-mediated bone remodeling model applied to a femur with subtrochanteric fracture: A subject-specific finite element study," Med. Eng. Phys., Jun. 2019.

2. E. M. Fortes et al., "[High morbid-mortability and reduced level of osteoporosis diagnosis among elderly people who had hip fractures in São Paulo City]," Arq. Bras. Endocrinol. Metabol., vol. 52, no. 7, pp. 1106-1114, Oct. 2008.

3. O. Johnell and J. Kanis, "Epidemiology of osteoporotic fractures," Osteoporos. Int. J. Establ. Result Coop. Eur. Found. Osteoporos. Natl. Osteoporos. Found. USA, vol. 16 Suppl 2, pp. S3-7, Mar. 2005.

4. S. Weiner and H. D. Wagner, "THE MATERIAL BONE: Structure-Mechanical Function Relations," Annu. Rev. Mater. Sci., vol. 28, no. 1, pp. 271-298, 1998.

5. A. Chennakesava Reddy and B. Kotiveerchari, "Simulation of Femur Bone Fracture in Car Accident using CT Scan Data and Finite Element Analysis,” Int. J. Sci. Res. IJSR, vol. 4, pp. 1805-1807, Nov. 2015.

6. J. E. Bertram and A. A. Biewener, "Bone curvature: sacrificing strength for load predictability?," J. Theor. Biol., vol. 131, no. 1, pp. 75-92, Mar. 1988.

7. T. P. Skuban, T. Vogel, A. Baur-Melnyk, V. Jansson, and B. Heimkes, "Function-orientated structural analysis of the proximal human femur," Cells Tissues Organs, vol. 190, no. 5, pp. 247-255, 2009.

8. M. Cuppone, B. B. Seedhom, E. Berry, and A. E. Ostell, "The longitudinal Young's modulus of cortical bone in the midshaft of human femur and its correlation with CT scanning data," Calcif. Tissue Int., vol. 74, no. 3, pp. 302-309, Mar. 2004.

9. F. Katsamanis and D. D. Raftopoulos, "Determination of mechanical properties of human femoral cortical bone by the Hopkinson bar stress technique," J. Biomech., vol. 23, no. 11, pp. 1173-1184, 1990.

10. T. D. Brown, M. E. Way, and A. B. Ferguson, "Mechanical characteristics of bone in femoral capital aseptic necrosis," Clin. Orthop., no. 156, pp. 240-247, May 1981.

11. Z.-F. Zhang, J.-L. Yang, H.-C. Jiang, Z. Lai, F. Wu, and Z.-X. Liu, "Updated association of tea consumption and bone mineral density: A meta-analysis," Medicine (Baltimore), vol. 96, no. 12, p. e6437, Mar. 2017.

12. Y. J. Yoon, "The effect of charge density on the velocity and attenuation of ultrasound waves in human cancellous bone," $J$. Biomech., vol. 79, pp. 54-57, 052018.

\section{AUTHORS PROFILE}

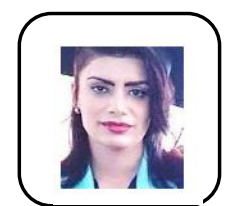

Houneida Sakly: Doctor and engineer in computer science. Currently, Researcher at the National School of Computer Science (ENSI) - COSMOS Laboratory and within the international medical Carthage Center in Tunisia.

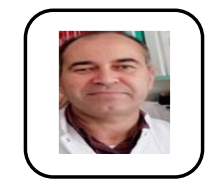

Mourad Said: Professor in Medical Imaging .Former doctor of Paris hospitals. A Member of the Middle East Africa Regional Committee and within the Radiological Society of North America (RSNA)

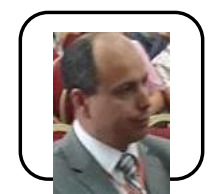

Moncef Tagina: Professor of High education with Responsibility of Research Master degree in Artificial Intelligence and decision support. Supervisor of $\mathrm{PhD}$ research project in the field of: IA and Robotics within the National School of Computer Science. in Tunisia 